\title{
Aquisição de intraverbais em crianças com autismo: efeitos do pareamento de estímulos e respostas ecoicas ${ }^{1}$
}

\author{
Malena Russelakis Carneiro Costa* (]) \\ Carlos Barbosa Alves de Souza
}

Universidade Federal do Pará, Núcleo de Teoria e Pesquisa do Comportamento. Belém, PA, Brasil

\begin{abstract}
Resumo: $O$ ensino de intraverbais para indivíduos com autismo tem sido realizado, principalmente, através de procedimentos que envolvem reforçamento diferencial. Recentemente, o procedimento de observação de pareamento de estímulos (SPOP) surgiu enquanto alternativa para verificar a emergência de repertórios verbais. O SPOP implica o pareamento de estímulos sem exigir outra resposta que a observação do pareamento. $\mathrm{O}$ ecoico tem sido apontado como facilitador na emergência de operantes verbais. O presente estudo comparou, com um delineamento de sondas múltiplas concorrentes com tratamento alternado adaptado, a eficácia do SPOP com um procedimento de apresentação contígua de estímulos com requisição de resposta ecoica na emergência de intraverbais em três crianças com autismo. Observou-se a eficácia do SPOP na indução de intraverbais e verificouse o papel facilitador do ecoico. Discutem-se possíveis efeitos de interferência dos tratamentos, da extinção de respostas no contexto experimental e da utilização de reforçadores condicionados como parte do SPOP.
\end{abstract}

Palavras-chave: procedimento de observação de pareamento de estímulos, intraverbais, ecoico, autismo.

\section{Introdução}

O transtorno do espectro autista (TEA) se caracteriza, entre outros aspectos, por dificuldade na comunicação social (American Psychiatric Association, 2013). Isto implica, nessa população, dificuldades com repertórios tais como conversação, respostas a perguntas e formação de sequências verbais (ex., narração de histórias) (Eikeseth \& Smith, 2013; Stauch, LaLonde, Plavnick, Bak, \& Gatewood, 2017). Esses repertórios podem ser caracterizados em termos do comportamento operante intraverbal (Skinner, 1957/1992).

Repertórios verbais têm sido ensinados para indivíduos com TEA principalmente através de procedimentos que envolvem reforçamento diferencial, tais como: ensino por tentativas realizadas em blocos (Haggar, Ingvarsson, \& Braun, 2018; Ingvarsson, Kramer, Carp, Pétursdóttir, \& Macias, 2016), procedimento com resposta de observação diferencial (Kisamore, Karsten, \& Mann, 2016) e procedimentos que

Endereço para correspondência:mal_russel@msn.com

1 Malena Costa recebeu bolsa de mestrado da Coordenação de Aperfeiçoamento de Pessoal de Nível Superior (Capes). Carlos Souza é bolsista de Produtividade do Conselho Nacional de Desenvolvimento Científico e Tecnológico (CNPq). A pesquisa contou com financiamento da Capes (Processo 88887091031201401) e do Instituto Nacional de Ciência e Tecnologia sobre Comportamento, Cognição e Ensino (CNPq - Processo 573972/2008-7, e Fundação de Amparo à Pesquisa do Estado de São Paulo - Processo 2008/57705-8).

2 Respostas verbais a estímulos antecedentes verbais com controle temático (não há correspondência ponto a ponto entre respostas e estímulos antecedentes), mantidas por reforçamento generalizado (Skinner, 1957/1992). objetivam comparar dicas vocais e visuais (Kodak, Fuchtman, \& Paden, 2012; Ingvarsson \& Hollobaugh, 2011; Vedora \& Conant, 2015; Vedora, Meunier, \& Mackay, 2009).

Um procedimento que começa a ser explorado no ensino de repertórios verbais é o procedimento de observação de pareamento de estímulos (SPOP, do inglês stimulus pairing observation procedure). Esse procedimento é caracterizado pela similaridade com o aprendizado em condições naturais e consiste na apresentação contígua de pares de estímulos (ex., uma palavra e um objeto), sem o reforçamento diferencial de repostas do participante. Posteriormente é verificado se houve a aprendizagem de relações entre os pares de estímulos (ex., o nome de um objeto) (Carnerero \& PérezGonzález; 2014; Rosales, Rehfeldt, \& Huffman, 2012).

$\mathrm{O}$ efeito positivo do SPOP tem sido observado na aquisição de leitura em crianças com desenvolvimento atípico (Omori \& Yamamoto, 2013), de soletração e escrita em crianças com autismo e com desenvolvimento típico (Omori \& Yamamoto, 2015), de tatos ${ }^{3}$ e respostas de ouvinte (seleção de objetos/figuras dados seus nomes) em crianças e adolescentes com autismo (Byrne, Rehfeldt, \& Aguirre, 2014; Carnerero \& Pérez-González, 2014) e com desenvolvimento típico (Pérez-González, Cereijo-Blanco, \& Carnerero, 2014; Pérez-González, García-Conde, \& Carnerero, 2011; Rosales et al., 2012). Três estudos avaliaram o efeito do SPOP sobre a aquisição de intraverbais (Carnerero \& Pérez-González,

3 Operante verbal controlado por estímulos antecedentes não verbais e mantido por reforço generalizado (Skinner, 1957/1992). 
2015; Carnerero, Pérez-González, \& Osuna, 2019; VallingerBrown \& Rosales, 2014).

Carnerero e Pérez-González (2015) investigaram a emergência de tatos, respostas de seleção (ouvinte) e intraverbais de instrumentos musicais e seus respectivos países em 12 participantes adultos com desenvolvimento típico. Os pareamentos realizados foram do tipo auditivoauditivo (som do instrumento - nome do instrumento; som do instrumento - nome do país do instrumento). Verificou-se que, após o SPOP, o repertório de tato emergiu em todos os participantes, e o de seleção emergiu na maioria. Já o intraverbal emergiu completamente em 3 dos 8 participantes, parcialmente em 4 dos 8 e não emergiu para 1 participante. Carnerero et al. (2019) replicaram Carnerero e Pérez-González (2015) e verificaram que testar primeiro o repertório de seleção após o SPOP resultou na aquisição de um número maior de intraverbais.

O estudo de Vallinger-Brown e Rosales (2014) foi o único, até o momento, que avaliou o efeito do SPOP na aprendizagem de intraverbais em crianças com TEA. $\mathrm{O}$ estudo comparou o SPOP com o "treino de ouvinte" para o ensino de intraverbais para três crianças com autismo com idades entre 4 e 7 anos (todas no nível 2 de intraverbal do Verbal behavior milestones assessment and placement program - VB-MAPP - Sundberg, 2008/2014; e uma no nível 3 e duas no nível 2 dos repertórios de mando ${ }^{4}$, tato e ouvinte). Foram utilizados dois conjuntos, cada um com seis estímulos auditivos (ex., "fica no topo da casa?") e seus correspondentes visuais (ex., figura de um telhado). As fases experimentais consistiram no treino direto de tato das figuras correspondentes ao estímulo-resposta, seguido de pré-teste de intraverbal, treino de utilização do iPad, implementação do SPOP e "treino de ouvinte" (realizados alternadamente), pós-teste de intraverbal e avaliação de generalização com novos experimentadores.

Eram realizadas 12 tentativas/pareamentos para o "treino de ouvinte" e SPOP. O "treino de ouvinte" iniciava com a exigência de uma resposta de observação (tocar em uma seta na tela do iPad), que apresentava seis estímulos visuais, e de um ícone de som na tela, que ao ser tocado pelo participante produzia uma instrução sonora relacionada com um dos estímulos visuais (ex., "Aponte para o que você usa para dizer a hora"). Respostas corretas eram reforçadas e as incorretas ensejavam dica gestual e outra oportunidade para responder. O SPOP se caracterizava pela exigência da resposta de observação, que apresentava um estímulo visual e um ícone de som na tela do iPad. Quando o participante tocava no ícone, produzia o estímulo auditivo relacionado ao estímulo visual que permanecia na tela (ex., "O relógio é usado para dizer a hora"). No SPOP era reforçada apenas a resposta de olhar para a tela. Os resultados demonstraram que o participante que estava predominantemente no nível 3 alcançou critério após

4 Operante verbal controlado por variáveis motivacionais e mantido por reforço específico (Skinner, 1957/1992).
10 pareamentos por estímulo no teste de intraverbal, e os outros dois participantes alcançaram critério de não aprendizagem após, em média, 27 pareamentos por estímulo. No entanto, outros estudos que utilizaram o SPOP para estabelecer tatos e respostas de ouvinte em crianças e adolescentes com TEA (Byrne et al., 2014; Carnerero \& Pérez-González, 2014; Rosales et al., 2012) mostraram que um número maior de pareamentos pode levar à aquisição dos repertórios verbais.

Vallinger-Brown e Rosales (2014) indicaram ainda uma relação entre a emissão de respostas ecoicas ${ }^{5}$ e a emergência dos intraverbais, uma vez que o participante que alcançou critério em ambos os procedimentos foi o que emitiu mais respostas ecoicas. Estudos utilizando o SPOP para emergência de classes de equivalência e operantes verbais também apontam esta correlação ou sugerem o comportamento ecoico enquanto pré-requisito (Carnerero \& Pérez-González, 2014; Rosales et al., 2012; Takahashi, Yamamoto, \& Noro, 2011 - mas ver Byrne et al., 2014). No entanto, nestes estudos a ocorrência do ecoico durante o pareamento não foi manipulada, sendo apenas registrada sua produção espontânea.

O ecoico é utilizado enquanto tipo de ajuda no ensino de intraverbais. Kodak et al. (2012) demonstraram que a dica ecoica com correção é mais eficiente em relação a dicas visuais, enquanto Vedora et al. (2009) e Ingvarsson e Hollobaugh (2011) demonstraram que dicas textuais e de tato são mais eficientes. Kisamore, Karsten, Mann e Conde (2013) e Kisamore et al. (2016) utilizaram um procedimento que consistia na repetição das partes relevantes da pergunta para o ensino de intraverbais e constataram que o comportamento ecoico funcionou como comportamento mediador, uma vez que, salientando os componentes relevantes do estímulo discriminativo, houve aumento da probabilidade de emissão da resposta correta. Estudos com crianças típicas (Coon \& Miguel, 2012) e com autismo (Vedora \& Conant, 2015) sugeriram que a eficiência do tipo de dica está relacionada com a história de reforçamento de cada sujeito com cada tipo de dica.

Dadas: (1) a importância de investigação do ecoico enquanto facilitador na emergência de intraverbais; (2) a escassez de estudos sobre o efeito do SPOP na aquisição deste operante verbal; (3) a dificuldade de aquisição de intraverbais por crianças com TEA e sua importância para o desenvolvimento de habilidades sociais e acadêmicas (Sundberg \& Sundberg, 2011); e (4) a importância de estabelecer repertórios socialmente relevantes como o aprendizado de segundo idioma (Cortez, Santos, Quintal, Silveira, \& de Rose, 2019; Pérez-González, Herszlikowicz, \& Williams, 2008), o presente estudo procurou comparar a eficácia do SPOP com um procedimento de apresentação contígua de estímulos (PACE) com requisição de resposta ecoica,

5 Operante verbal controlado por antecedente verbal vocal, com correspondência ponto a ponto entre antecedente e resposta (Skinner, 1957/1992). 
na emergência de intraverbais em crianças com TEA. Além disso, procurou-se homogeneizar o repertório de entrada dos participantes de forma que todos estivessem predominantemente no mesmo nível da avaliação do VB-MAPP e avaliar os efeitos dos procedimentos na manutenção do repertório ao longo do tempo e na generalização entre pessoas.

\section{Método}

\section{Participantes}

Participaram do estudo três crianças do sexo masculino (P1: 4 anos, P2: 5 anos e P3: 7 anos). Os participantes $\mathrm{P} 1$ e $\mathrm{P} 3$ recebiam intervenção analíticocomportamental três vezes por semana durante duas horas no Projeto Atendimento e Pesquisa sobre Aprendizagem e Desenvolvimento (APRENDE) da Universidade Federal do Pará (UFPa) e P2 recebia intervenção analítico-comportamental em uma clínica privada. Todos os participantes tinham diagnóstico de TEA. Os repertórios verbais dos participantes foram avaliados pelo instrumento do VB-MAPP (Sundberg, 2008/2014). Os participantes P1 e P2 apresentavam habilidades intraverbais condizentes com o $7^{\circ}$ marco (7M) do desenvolvimento (nível $2-18$ a 30 meses), referente à habilidade de responder ao primeiro nome quando perguntado "qual seu nome?" e preencher lacunas (ex., "um, dois, três e? Já", "seu Lobato tinha um sítio? Iaiaô"), e P3 pontuava até o 7M, mas com alguns intraverbais de preenchimento de lacunas que não envolviam músicas ou brincadeiras sociais (pontuava $1 / 2$ no $8 \mathrm{M}$ ).

Os participantes $\mathrm{P} 1$ e $\mathrm{P} 2$ apresentavam repertório de tato condizente com o nível 2 , pontuando até $6 \mathrm{M}$, referente à habilidade de tatear 25 itens quando perguntados "o que é isso?", e P3 pontuava até 7M, que corresponde a generalizar tatos por meio de três exemplares de 50 itens testados. Quanto ao repertório de ouvinte, P1 e P3 pontuavam até $8 \mathrm{M}$ (nível 2), correspondente à habilidade de realizar 10 ações motoras específicas sob comando, $\mathrm{P} 2$ pontuava até $5 \mathrm{M}$ (nível 1), conseguindo selecionar os itens corretos de um conjunto de quatro itens para 20 diferentes objetos ou figuras.

Os participantes não emitiam comportamentos auto e heterolesivos que concorressem com a implementação do procedimento. Salienta-se que P1 não tinha história de ensino de intraverbais anterior à implementação desse procedimento.

Os responsáveis legais dos participantes assinaram um termo de consentimento livre e esclarecido autorizando a participação no estudo, que foi aprovado pelo comitê de ética em pesquisa do Núcleo de Medicina Tropical da UFPa (Parecer 3.172.492).

\section{Ambiente, material e equipamentos}

A coleta de dados foi conduzida nas instalações do Projeto APRENDE, localizado no Núcleo de Teoria e Pesquisa do Comportamento da UFPa, ou na casa dos participantes quando não havia possibilidade de deslocamento até as instalações do projeto. No APRENDE as sessões experimentais foram realizadas em uma sala (de $5 \mathrm{~m} \times 2,5 \mathrm{~m})$ climatizada e equipada com mobiliário adequado para atividades com crianças.

Para fins de controle metodológico, as condições ambientais na casa do participante foram arranjadas de forma que o ambiente fosse similar ao do laboratório. Portanto, na casa dos participantes as sessões foram realizadas em um cômodo climatizado com brinquedos, uma mesa e duas cadeiras infantis, com iluminação natural e artificial, no qual ficavam somente o participante e o experimentador durante as sessões.

Para o registro e coleta dos dados foi utilizada uma câmera Sony DCR-SX22, lápis, folhas de registro e estímulos impressos desenvolvidos para o experimento.
Conjunto 1

Machado em inglês é?
Conjunto 2

Bomba em inglês é?

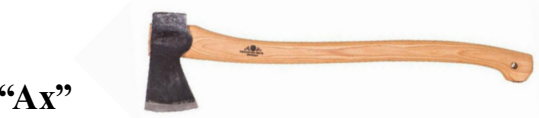

"Ax"

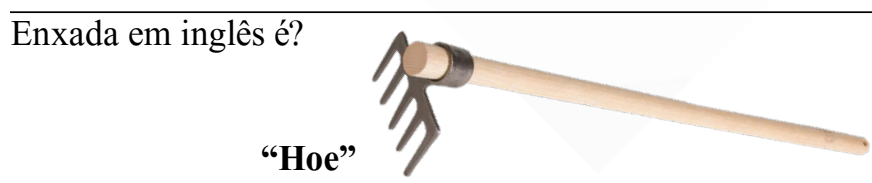

Forno em inglês é?

Serra em inglês é?

"Saw"

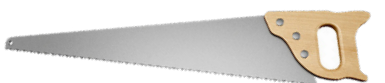

Tubo em inglês é?

"Pipe"

Figura 1. Conjuntos de estímulos utilizados no estudo. 


\section{Estímulos}

Foram utilizados dois conjuntos com três estímulos cada, formados por estímulos auditivos e visuais. Os estímulos visuais foram figuras coloridas (ex., fotos de um machado, enxada, serra etc.) impressas em cartões de $10 \times 10 \mathrm{~cm}$, e os estímulos auditivos foram frases faladas pelo experimentador relacionadas com as figuras (ex., "machado em inglês é?", "serra em inglês é?") (ver Figura 1). Foram selecionadas figuras cujos nomes em inglês a criança conseguia ecoar em uma avaliação realizada na fase 1 do procedimento (preferencialmente palavras monossílabas ou dissílabas).

\section{Estímulos consequentes}

Para selecionar possíveis estímulos reforçadores foi realizado um levantamento prévio com os cuidadores das crianças e, no caso daquelas recebendo intervenção comportamental, com as equipes responsáveis. Também houve consequências na forma de elogios (por exemplo, "muito bem", "você acertou" etc.).

\section{Delineamento experimental}

Foi utilizado o delineamento de sondas múltiplas concorrentes entre participantes (Horner \& Baer, 1978) com tratamento alternado adaptado (Sindelar, Rosenberg, \& Wilson, 1985), evitando os efeitos de uma longa exposição a sessões de linha de base e expondo o participante a dois tipos de tratamentos, SPOP sem exigência de resposta ecoica (tratamento A) e o PACE com exigência de reposta ecoica (tratamento B), para comparar a eficácia desses tratamentos.

Para controlar possíveis efeitos de complexidade dos estímulos, a apresentação dos conjuntos de estímulos foi balanceada nos tratamentos entre os participantes: para P1 e P3 foi apresentado o conjunto 1 no tratamento A e o conjunto 2 no tratamento B. Para P2 essa ordem foi invertida. Visando controlar possíveis efeitos de ordem de apresentação dos tratamentos, a implementação deles também foi balanceada entre as sessões.

Em uma adaptação do delineamento de sondas múltiplas entre participantes, caso os participantes não apresentassem uma tendência crescente nos testes de intraverbal após 45 pareamentos por estímulo (equivalente a 9 ciclos) ou não alcançassem critério após 60 pareamentos por estímulo (equivalente a 12 ciclos), o tratamento era inserido para o próximo participante, de forma que estes não ficassem sem intervenção por períodos prolongados.

\section{Variável independente e dependente}

As variáveis independentes foram os ciclos de SPOP-teste e o PACE com exigência de resposta ecoica-teste. Considerava-se um acerto quando a criança respondia vocalmente às relações especificadas na Figura 1 (ex., responder "ax" na presença do estímulo discriminativo "machado em inglês é?"). A variável dependente foi a porcentagem de respostas corretas nos testes de intraverbal após os tratamentos.

\section{Procedimento}

O procedimento consistiu em sete fases: (1) avaliação da articulação das palavras; (2) sonda de intraverbal; (3) treino de tato; (4) linha de base; (5) implementação dos tratamentos; (6) teste de generalização; e (7) teste de manutenção.

\section{Fase 1: Avaliação da articulação das palavras}

Foram pré-selecionadas 20 palavras monossílabas ou dissílabas em inglês (ex. pump, pipe, ax, hoe etc.) e realizadas sessões de teste de ecoico desses nomes. Cada sessão constituiu-se de 20 tentativas (uma para cada estímulo). Em cada tentativa, o pesquisador dizia uma palavra e solicitava que a criança a repetisse. Não houve consequência para acerto ou erro. Após a reposta da criança, ou caso ela não emitisse resposta em até cinco segundos, o pesquisador iniciava a tentativa seguinte com uma palavra diferente. A cada duas ou três tentativas eram realizadas tentativas de respostas que a criança já respondia sem dificuldade (tentativas de manutenção), possibilitando o acesso a reforçadores. Esse procedimento de reforçamento de respostas já estabelecidas, intercaladas com as tentativas experimentais (sondas, SPOP, PACE e testes), foi mantido nas restantes fases do procedimento.

As sessões foram realizadas até que se obtivessem, pelo menos, nove palavras cujos nomes em inglês todas as crianças reproduzissem corretamente (para contar com três estímulos adicionais para eventuais necessidades de substituição de estímulos - os estímulos utilizados encontram-se na Figura 1).

\section{Fase 2: Sonda de intraverbal}

Foi realizada uma sonda inicial ante as possíveis relações dos conjuntos 1 e 2, em um bloco com nove tentativas (três para cada estímulo) para cada conjunto. Cada tentativa consistiu na obtenção de atenção do participante, seguida da apresentação da pergunta pelo experimentador (ex., "machado em inglês é?"), espera pela resposta do participante em até três segundos. Respostas, corretas ou incorretas, não foram consequenciadas (e foram intercaladas com tentativas de manutenção - ver Fase 1). $\mathrm{O}$ intervalo entre tentativas foi de aproximadamente três segundos entre um antecedente e outro. O critério para que o participante seguisse no estudo era que ele não emitisse corretamente a nenhum antecedente testado (ex., "machado em inglês é?"). 


\section{Fase 3: Treino de tato}

O treino de tato foi conduzido com os estímulos visuais dos conjuntos 1 e 2 , assegurando que os participantes poderiam tatear todos os estímulos utilizados no procedimento, e visando a ocorrência de controle conjunto (Lowenkron, 1998) entre o estímulo antecedente verbal e o estímulo visual durante a etapa de SPOP dos tratamentos A e B, favorecendo a transferência de controle do estímulo visual para o auditivo nos testes intraverbais.

O treino foi conduzido com um estímulo por vez, em blocos com cinco tentativas, e quando o participante apresentava $80 \%$ de respostas corretas para o tato do estímulo em treino, era adicionado outro estímulo. Esse processo continuou até que todos os estímulos foram treinados em um bloco com 30 tentativas (cinco apresentações de cada estímulo) randomizadas. A tentativa consistia na obtenção da atenção do participante, apresentação da figura pelo experimentador, seguida da pergunta “o que é isso?". Respostas corretas (em inglês) foram reforçadas diferencialmente através de elogio seguido de item de preferência da criança. Respostas incorretas eram corrigidas, na forma de dica ecoica imediata e outra oportunidade para responder. Caso o participante não emitisse resposta ou emitisse uma resposta incorreta na nova oportunidade para responder, a correção era repetida no máximo três vezes e passava-se para a próxima tentativa. Ao longo do bloco de tentativas, as dicas ecoicas foram esvanecidas gradualmente para dicas ecoicas parciais (ex., "a" para "ax") e parciais com atraso de 2 segundos (ex., espera de 2 segundos antes da dica parcial "a" para palavra "ax") até que o participante emitisse respostas corretas de forma independente. $\mathrm{O}$ critério de aprendizagem para que o participante pudesse passar para a fase seguinte era de uma sessão com, pelo menos, $96 \%$ de respostas corretas em um bloco.

\section{Fase 4: Linha de base}

Após o critério no treino de tato, era realizada a linha de base, que consistiu em sessões de sondas de intraverbal com os estímulos dos conjuntos 1 e 2 , idênticas às da Fase 2, até que o participante demonstrasse estabilidade no desempenho (oscilação igual ou inferior a 5\% no percentual de acertos entre sessões) ou uma tendência decrescente de acertos, considerando, pelo menos, três sessões.

\section{Fase 5: Implementação dos tratamentos}

A implementação dos dois tratamentos foi realizada de forma alternada (Tratamento A - ciclos de SPOP-teste de intraverbal; Tratamento B - PACE com exigência de resposta ecoica-teste de intraverbal), um com cada conjunto de estímulos. Foram realizadas, no mínimo, três sessões por semana, e um a três ciclos por sessão. No Tratamento
A, a etapa de SPOP dos ciclos teve 15 pareamentos (cinco para cada estímulo - considerando o número de pareamentos por ciclo nos estudos de Byrne et al., 2014, e Carnerero \& Pérez-González, 2014). O pareamento consistiu na apresentação do estímulo auditivo (ex., frase falada "machado em inglês é?") enquanto o experimentador apresentava a figura correspondente à resposta (ex., figura do machado). O intervalo de tempo entre um pareamento e outro foi de, aproximadamente, três segundos, e os pareamentos eram randomizados. O teste das respostas intraverbais foi realizado dois minutos após a finalização dos 15 pareamentos. Este consistiu em nove tentativas (três para cada estímulo), também randomizadas, e foi realizado de forma idêntica à sonda de intraverbal (Fase 2).

O Tratamento B (ciclos de PACE com exigência de resposta ecoica e teste) foi realizado de forma idêntica ao Tratamento $\mathrm{A}$, com a diferença de que após o PACE (estímulo visual e auditivo), em três dos cinco pareamentos de cada estímulo, o experimentador imediatamente fornecia a dica ecoica (ex., "ax") e, caso a criança não ecoasse a dica, ele solicitava que o participante repetisse ("Diga __", "Repita _."); caso a criança ecoasse, o experimentador registrava a emissão do comportamento e, caso ela não ecoasse, seguia-se para o próximo pareamento. As eventuais respostas ecoicas não eram consequenciadas. As respostas ecoicas eram exigidas em três das cinco apresentações, visando evitar efeitos de extinção da resposta, uma vez que estas não eram reforçadas.

Os ciclos, tanto no Tratamento A quanto no Tratamento B, foram realizados até que: (1) o desempenho do participante no teste fosse de $89 \%$ de acertos (oito acertos em nove tentativas) em uma sessão, ou até que (2) fossem realizados 255 pareamentos por estímulo (51 ciclos), considerando o máximo de pareamentos necessário para a aquisição de tatos e resposta de ouvinte no estudo de Byrne et al. (2014). O intervalo entre a realização de um ciclo e outro foi de, no mínimo, cinco minutos. Caso o desempenho do participante alcançasse o critério de aprendizagem nos testes, ele era exposto na sessão seguinte à Fase 6 para verificar a generalização da aprendizagem com outras pessoas, e quatro semanas depois da Fase 7 para avaliar a manutenção da aprendizagem. Caso o desempenho do participante não alcançasse critério de aprendizagem nos testes após 255 pareamentos (51 ciclos), sua participação no estudo era encerrada e ele era encaminhado para o ensino direto das relações intraverbais como parte da intervenção analítico-comportamental à qual ele estava sendo exposto.

\section{Fase 6: Teste de generalização}

Alcançado o critério de aprendizagem nos tratamentos A e/ou B, o teste de generalização foi conduzido de forma idêntica à Fase 2, porém com experimentadores que não faziam parte do ambiente natural do participante. 


\section{Fase 7: Teste de manutenção}

O teste de manutenção foi conduzido de forma idêntica aos ciclos da Fase 5, transcorridas quatro semanas.

\section{Registro e análise de dados}

Nas sessões de sonda, linha de base e testes foram registradas as respostas corretas e incorretas para as relações intraverbais. Também foram registradas as respostas vocais espontâneas emitidas durante os pareamentos visuaisauditivos no tratamento sem exigência de resposta ecoica, e as respostas ecoicas durante o tratamento que as exigia, procurando avaliar possíveis relações entre estas respostas e o desempenho nos testes de intraverbal. Foi analisada a porcentagem de respostas corretas nas sondas, linha de base e testes de intraverbal em ambos os tratamentos, visando comparar a efetividade dos tratamentos e o número de ciclos necessários para o alcance de critério, de forma a comparar a eficiência dos dois tratamentos.

\section{Concordância entre observadores e integridade do procedimento}

A partir dos vídeos das sessões experimentais, outro pesquisador fez o registro do desempenho de cada participante em $30 \%$ de cada etapa do estudo, visando avaliar a fidedignidade do registro do desempenho dos participantes. Com base na comparação de ambos os registros, tentativa a tentativa, foi calculado o índice de concordância entre observadores ([concordância/ concordância +discordância] $\times 100$ ). A concordância entre observadores nos registros do desempenho dos participantes em todas as fases do procedimento foi de $100 \%, 98 \%$ e $98 \%$ para $\mathrm{P} 1, \mathrm{P} 2$ e $\mathrm{P} 3$, respectivamente.

Através dos vídeos das sessões experimentais foi realizada uma avaliação da precisão de aplicação dos procedimentos por um avaliador externo à pesquisa para $30 \%$ das sessões de cada fase do procedimento para cada participante. Esta avaliação foi feita através de um checklist de integridade do procedimento em cada fase, no qual especificou-se o que conta como uma implementação correta do Tratamento A, descritas como: (1) garantir atenção do participante, (2) realização do pareamento auditivo-visual, (3) não consequenciar quaisquer tipos de resposta em ambos os tratamentos, e (4) requisição de resposta ecoica (apenas para o tratamento B). O cálculo da integridade foi realizado da seguinte forma: [implementações corretas/ total de implementações] $\times 100$. A média da integridade de todas as fases do procedimento resultou em $99 \%, 99 \% \mathrm{e}$ $100 \%$ para $\mathrm{P} 1, \mathrm{P} 2$ e $\mathrm{P} 3$, respectivamente.

\section{Resultados}

Todos os participantes alcançaram critério de aprendizagem dos tatos em inglês dos estímulos dos conjuntos 1 e 2 - P1 em 24 blocos de treino, P2 em 42, e P3 em 29. A Figura 2 apresenta o número de respostas intraverbais corretas dos participantes nas sondas, linhas de base, implementação dos tratamentos e testes de generalização e manutenção.

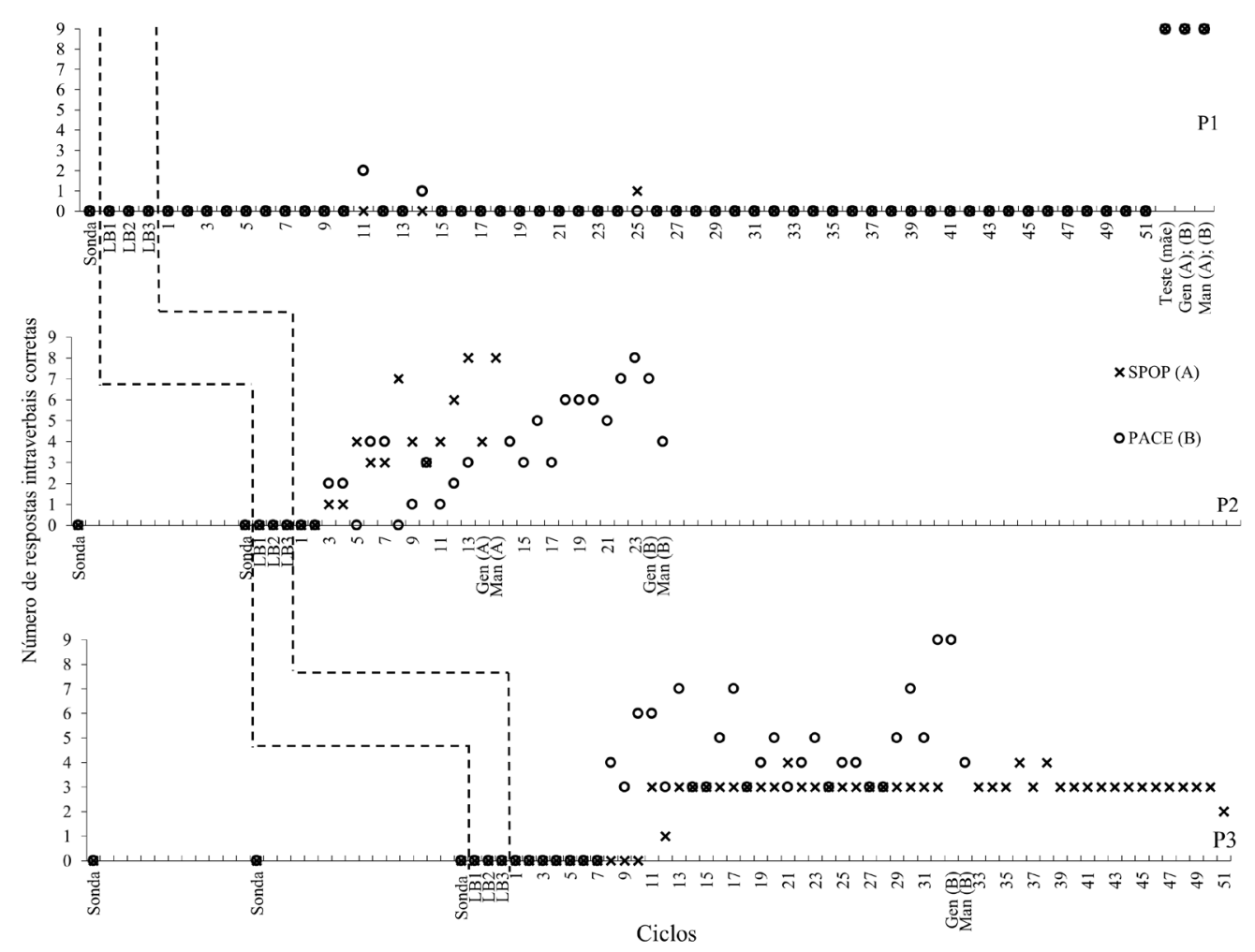

Figura 2. Número de respostas intraverbais corretas dos participantes nas sondas, linhas de base, implementação dos tratamentos, generalização (Gen) e manutenção (Man) 

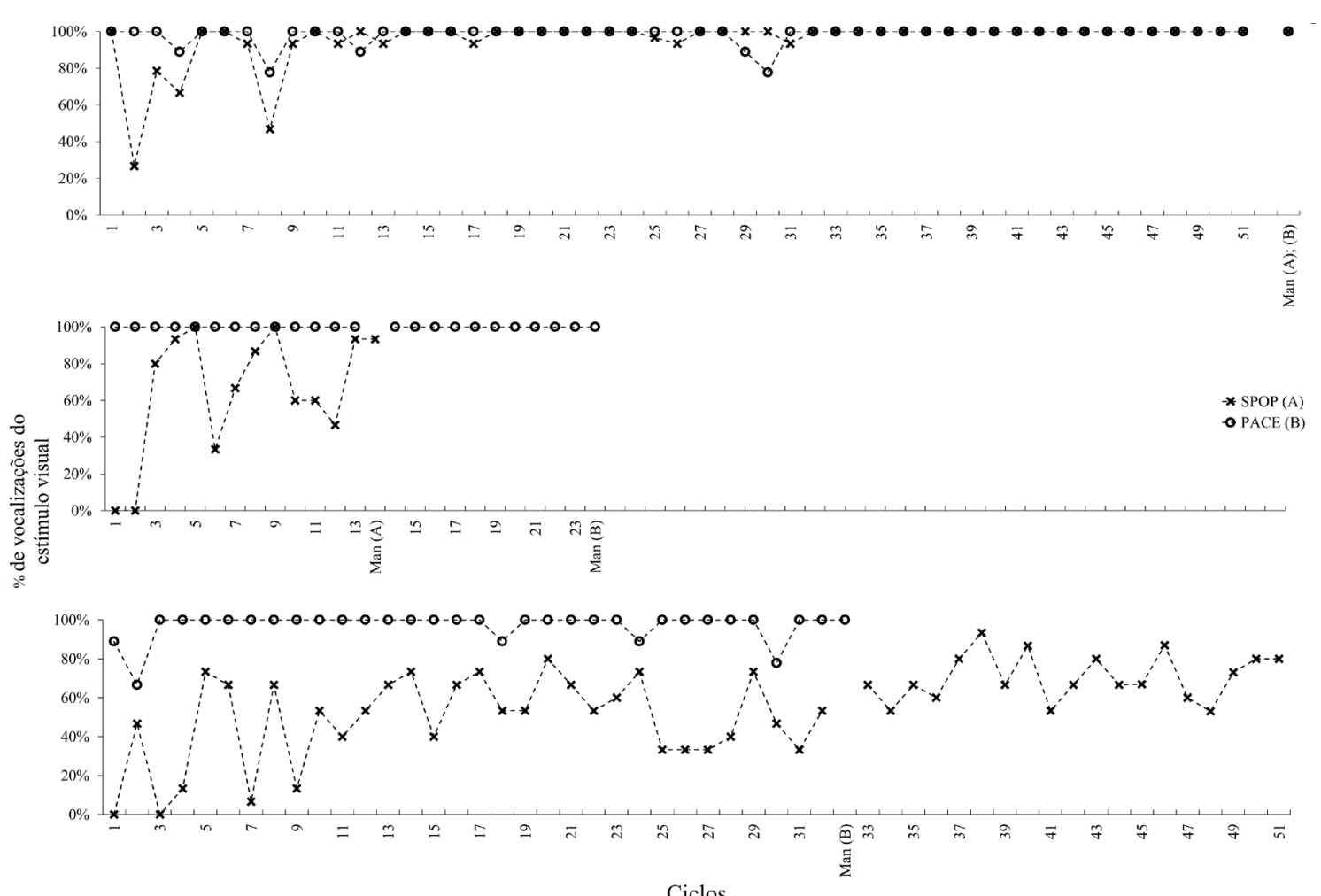

Figura 3. Percentual de vocalizações do estímulo visual emitidas pelos participantes durante a implementação dos tratamentos e nos testes de manutenção (Man)

Observa-se que nenhum dos participantes emitiu respostas corretas nas sondas ou linha de base. O participante P2 alcançou o critério de aprendizagem dos intraverbais durante a implementação de ambos os tratamentos: em 13 ciclos no tratamento A, e em 23 no tratamento B. Nos testes de generalização, o desempenho de P2 ficou abaixo do critério de aprendizagem: $44 \%$ e $78 \%$ de acertos para os tratamentos A e B, respectivamente. Nos testes de manutenção, $\mathrm{P} 2$ alcançou o critério de aprendizagem $(89 \%)$ no tratamento A, mas não no tratamento B (44\%).

O participante $\mathrm{P} 3$ atingiu o critério de aprendizagem apenas com o conjunto de estímulos utilizado no tratamento B: no $32^{\circ}$ ciclo com $100 \%$ de respostas corretas. No teste de generalização com esse conjunto, o desempenho de $\mathrm{P} 3$ foi de $100 \%$ de respostas intraverbais corretas, e na manutenção seu desempenho foi de $44 \%$ de respostas corretas. No tratamento A, o desempenho de $\mathrm{P} 3$ variou entre $33 \%$ e $44 \%$ de respostas intraverbais corretas ao longo de 51 ciclos, demonstrando aprendizagem apenas de um intraverbal do conjunto ("machado em inglês é? Ax").

O participante P1 não alcançou critério de aprendizagem durante a implementação de nenhum dos tratamentos, com a emissão de algumas respostas corretas nos $11^{\circ}, 14^{\circ}$ e $25^{\circ}$ ciclos. No entanto, com o decorrer da implementação, a cuidadora relatou para a experimentadora que o participante emitia as relações intraverbais (ex., "machado em inglês é ax") fora do ambiente experimental. Com base nessa informação e no conhecimento sobre a função discriminativa/reforçadora que a mãe/cuidadora exerce desde cedo no desenvolvimento infantil (Greer, Pistoljevic, Cahill, \& Du, 2011; Longano \& Greer, 2015; Petursdottir \& Lepper, 2015; Petursdottir, Carp, Matthies, \& Esch, 2011), uma vez finalizada a implementação dos 51 ciclos previstos para os casos nos quais os participantes não alcançassem o critério de aprendizagem, foi realizada uma sessão de teste de intraverbal aplicado pela cuidadora no ambiente experimental (sob supervisão da experimentadora) ${ }^{6}$. Neste teste (teste (mãe) na Figura 2), P1 apresentou $100 \%$ de respostas intraverbais corretas nas relações de ambos os tratamentos. Esse desempenho se manteve nos testes de generalização e manutenção (este último realizado também pela cuidadora).

A Figura 3 apresenta o percentual de vocalizações dos estímulos-resposta (tais respostas poderiam ser classificadas funcionalmente enquanto tatos e intraverbais no tratamento A, e tatos, intraverbais e ecoicos no tratamento B) emitidas pelos

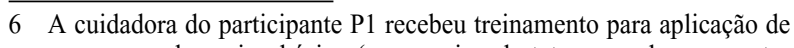
programas de ensino básico (e.g., ensino de tatos, mandos, respostas de imitação) por tentativas discretas como parte da intervenção comportamental à qual $\mathrm{P} 1$ era exposto fora do contexto experimental. Ao realizar os testes do referido procedimento, o fez de forma idêntica à experimentadora. 
participantes na implementação dos tratamentos e nos testes de manutenção. Todos os participantes apresentaram respostas durante a implementação de ambos os tratamentos, com um maior percentual de ocorrência no tratamento B. O participante P1 emitiu uma média de $96 \%$ de respostas no tratamento A e $99 \%$ durante o tratamento B. Para o participante P2 a média foi de $65 \%$ de respostas durante o tratamento A e $100 \%$ no tratamento B, e para $\mathrm{P} 3$ foi de $56 \%$ no A e $97 \%$ no B.

\section{Discussão}

Este estudo buscou comparar a eficácia do SPOP com um PACE com exigência de resposta ecoica na emergência de intraverbais em crianças com TEA. Procurou ainda estender os ciclos em ambos os tratamentos para avaliar o efeito de um número maior de pareamentos sobre a emergência dos intraverbais. Os resultados demonstraram a eficácia do SPOP e do PACE com exigência de resposta ecoica para induzir a emergência de intraverbais para crianças diagnosticadas com TEA: todos os participantes alcançaram critério de aprendizagem com o tratamento em que era requerida resposta ecoica, e dois participantes ( $\mathrm{P} 1$ e $\mathrm{P} 2$ ) alcançaram critério de aprendizagem no SPOP. Esses resultados se alinham com os de Vallinger-Brown e Rosales (2014), que verificaram a eficácia do SPOP na indução de intraverbais em um participante com TEA.

$\mathrm{O}$ fato de que todos os participantes alcançaram critério de aprendizagem com o conjunto de estímulos utilizado no tratamento $B$ sugere um efeito facilitador da inclusão de exigência de respostas ecoicas ao se utilizar procedimentos caracterizados pelo PACE para induzir a emergência de intraverbais. Esse resultado corrobora achados de outros estudos que registraram a ocorrência de ecoicos durante a implementação de SPOP para induzir repertórios verbais em crianças com desenvolvimento típico e atípico (Carnerero \& Pérez-González, 2014; Rosales et al., 2012; Takahashi et al., 2011; VallingerBrown \& Rosales, 2014).

No entanto, considerando que no tratamento A (SPOP) todos os participantes emitiram respostas vocais, em maior ou menor proporção, referentes ao estímulo visual (P1 de forma mais consistente e P3 de maneira mais reduzida), e que os participantes P1 e P2 também alcançaram o critério de aprendizagem nesse tratamento, a avaliação da eficácia do SPOP não foi conclusiva no presente estudo. Ainda que P3 não tenha alcançado critério de aprendizagem no tratamento sem requisição de resposta ecoica, seria inadequado considerar esse tratamento ineficaz ou menos eficiente que o tratamento com requisição de resposta ecoica, considerando os desempenhos dos participantes P1 e P2 (especialmente o desempenho de $\mathrm{P} 2$, que alcançou critério de aprendizagem primeiro no tratamento A).
Novos estudos que procurem avaliar o papel das repostas ecoicas na aprendizagem de respostas intraverbais, e de outros operantes verbais, devem atentar para os resultados de estudos recentes (Cao \& Greer, 2019; Longano \& Greer, 2015) que apontam para a necessidade de que estímulos visuais (em especial a presença de outras pessoas) e auditivos (em especial as vocalizações de outras pessoas) tenham sido estabelecidos como estímulos discriminativos/ reforçadores condicionais, visando verificar a frequência na emissão de respostas ecoicas e sua relação com a emergência de repertórios verbais (Horne \& Lowe, 1996). Por exemplo, Longano e Greer (2015) demonstraram que o condicionamento dos estímulos visuais e auditivos enquanto reforçadores esteve relacionado com aumento na emissão de ecoicos durante a implementação do procedimento com múltiplas instruções, resultando na emergência de nomeação bidirecional em duas crianças com autismo e uma com baixo desempenho escolar. Cao e Greer (2019) demonstraram que o treino intensivo de ecoico de palavras em chinês para crianças monolíngues inglesas (com desenvolvimento típico e atípico) facilitou a emergência de nomeação bidirecional de itens familiares e não-familiares em chinês, corroborando, de acordo com os autores, a noção de que a paridade entre o som escutado e reproduzido funcionaria como reforçador do comportamento de falante (Palmer, 1996).

Diante do exposto, é possível que a emissão de respostas ecoicas dos participantes do presente estudo, mesmo que não reforçada diretamente, tenha sido reforçada pela paridade entre o estímulo auditivo emitido pelo experimentador e a correspondência emitida pelos participantes (Palmer, 1996). Esta seria uma possível explicação de porque elas continuaram sendo emitidas em alta frequência pelos participantes no tratamento com requisição de resposta ecoica e variando no tratamento sem requisição de resposta ecoica. Além disso, pode ter ocorrido controle conjunto (Lowenkron, 1998) durante a implementação dos tratamentos, considerando que os participantes ecoavam o estímulo auditivo fornecido pelo experimentador na presença do estímulo visual durante o pareamento. Ainda assim, a seleção de estímulos com função reforçadora poderia ter favorecido ainda mais a paridade e o controle conjunto de estímulos, processos comportamentais que podem ser relevantes para a aquisição de repertórios verbais (Greer \& Longano, 2010).

No que concerne à relação entre a quantidade de ciclos de pareamentos-teste e o desempenho dos participantes, no estudo atual verificou-se que foram necessários 65 e 115 pareamentos por estímulo para que $\mathrm{P} 2$ atingisse critério de aprendizagem nos tratamentos $\mathrm{A}$ e $\mathrm{B}$, respectivamente. $\mathrm{O}$ participante $\mathrm{P} 3$ atingiu critério após 160 pareamentos por estímulo para o tratamento B. Com relação ao participante $\mathrm{P} 1$, o número não pôde ser calculado, uma vez que ele emitiu as relações intraverbais fora do ambiente experimental, e fazer afirmações sobre número de pareamentos não seria prudente neste caso. 
A quantidade de pareamentos realizada aproxima-se do número máximo de pareamentos necessários para aprendizagem dos repertórios de tato e ouvinte encontrados nos estudos de Byrne et al. (2014) e Carnerero e Pérez-González (2014), indicando que o número de pareamentos no SPOP pode ser uma variável relevante na determinação de sua eficácia.

O desempenho do participante P1 foi particularmente interessante, visto que este passou a emitir, de acordo com a cuidadora, os intraverbais fora do ambiente experimental, levando ao teste (bemsucedido) com ela no ambiente experimental após a finalização dos ciclos de pareamento previstos. $\mathrm{O}$ baixo desempenho de P1 com os experimentadores pôde estar relacionado inicialmente com uma incompreensão da tarefa, considerando que ele não tinha história prévia de ensino de intraverbais no contexto da intervenção geral da qual ele participava no APRENDE. Considerando esta falta de contextualização da tarefa intraverbal exigida e o fato de que durante os tratamentos as respostas dos participantes não eram reforçadas, estas podem ter sido colocadas em extinção no contexto do ambiente experimental.

Quando os testes foram realizados pela cuidadora, P1 demonstrou $100 \%$ de respostas intraverbais corretas com os conjuntos de estímulos utilizados em ambos os tratamentos. Este desempenho pode estar relacionado com a função discriminativa/reforçadora que a voz/presença da mãe/cuidadores pode adquirir desde muito cedo no desenvolvimento da criança (Greer et al., 2011; Longano \& Greer, 2015; Petursdottir \& Lepper, 2015; Petursdottir et al., 2011). A criança aprende a discriminar a forma como os cuidadores falam com ela através de características como velocidade da fala, repetição de nomes e instruções, acentuação de nomes e entonações mais exageradas, o que torna o discurso e a presença do cuidador, por conseguinte, mais saliente (DeCasper \& Fifer, 1980; Fernald, 1993), e ambos relacionados com ocasião para a emissão de comportamentos socialmente relevantes e obtenção de reforços (Greer \& Longano, 2010; Horne \& Lowe, 1996). Logo, a emissão de respostas intraverbais corretas com a cuidadora e não com os experimentadores pode ter ocorrido em função de uma história de extinção ocorrida com estes últimos e de história de reforçamento bem estabelecida com aquela.

Uma das limitações do atual estudo diz respeito ao possível efeito de interferência (carry-over effect) do tratamento com requisição de resposta ecoica sobre o tratamento sem exigência. Todos os participantes emitiriam vocalizações espontâneas dos estímulosresposta no tratamento sem exigência de respostas ecoicas. É possível que a alternância da condição na qual a resposta ecoica era exigida com aquela na qual ela não era exigida tenha terminado por induzir as vocalizações nessa última condição, impedindo a comparação da implementação do SPOP com o PACE com exigência de resposta ecoica. Uma forma de contornar essa dificuldade em novos estudos pode ser empregar um delineamento que implemente primeiro um tratamento, até o critério de aprendizagem/ interrupção, e depois o outro, com os adequados controles experimentais.

Outra limitação está relacionada à não realização de uma avaliação formal de preferência de reforçadores antes de cada sessão experimental. Os reforçadores utilizados nas sessões foram selecionados a partir do relato dos pais e terapeutas das crianças. Apesar do procedimento implementado não fornecer reforçamento diferencial para quaisquer respostas relacionadas aos pareamentos, a avaliação formal de reforçadores a cada sessão poderia ter contribuído para emissão de comportamento mais colaborativos dos participantes ao longo do estudo. Ainda no que diz respeito à função reforçadora dos estímulos, no presente estudo não foi analisado previamente a possível função reforçadora dos estímulos utilizados. Talvez o procedimento tivesse sido mais eficiente caso o experimentador tivesse se assegurado de que os estímulos auditivos e visuais funcionassem enquanto reforçadores.

Apesar dessas limitações, o presente estudo colaborou para ampliar o conhecimento sobre os efeitos do SPOP e o efeito da exigência de resposta ecoica em um PACE para a indução de repertórios verbais em indivíduos com TEA, além de estender o entendimento sobre aprendizagem de segundo idioma nessa população. Considerando que o intraverbal é um repertório de suma importância para o desenvolvimento de habilidades acadêmicas, sociais e de comunicação (Sundberg \& Michael, 2001), a investigação de procedimentos que facilitem sua aprendizagem é essencial, principalmente através de procedimentos que se assemelhem ao aprendizado em situações naturais, como é o caso do SPOP. Esta pesquisa também ajudou a ampliar o estudo de aprendizagem de segundo idioma e a efetividade do SPOP e da exigência de resposta ecoica na aquisição desse repertório.

\section{Intraverbal acquisition in autistic children: effects of stimulus pairing and echoic responses}

\footnotetext{
Abstract: Intraverbals have been taught to individuals with autism primarily through procedures involving differential reinforcement. Recently, Stimulus Pairing Observation Procedure (SPOP) emerged as an alternative to verify the emergence of verbal repertoires. The SPOP consists in the simultaneous presentation of two stimuli and requires only the observation response by the participant. The echoic behavior had been pointed out as a mediator in the emergence of verbal operants.
} 
This study compared the efficacy of the SPOP and of a contiguous stimulus presentation procedure with an echoic response requirement in emergence of intraverbals in three children with autism by using a concurrent multiple probe design with an adapted alternating treatment. The results showed the SPOP's efficacy for intraverbals induction and suggested a facilitating role of the echoic. A possible carry-over effect between treatments, extinction in the experimental context and the use of conditioned reinforcers in SPOP are discussed.

Keywords: stimulus pairing observation procedure, intraverbal, echoic, autism.

\section{Acquisition du comportement intraverbal chez des enfants autistes : effets de l'appariement des stimuli et des réponses échoïques}

Résumé : L'enseignement de l'intraverbal chez des individus autistes a été principalement réalisé avec des procédures impliquant le renforcement différentiel. Plus récemment, la Procédure d'Observation de Paires des Stimuli (SPOP en anglais) s'est imposée comme alternative pour vérifier l'émergence de répertoires verbaux. Le SPOP implique l'appariement des stimuli sans exiger d'autre réponse que l'observation des paires. Par ailleurs, l'échoïque a été signalé comme facilitateur de l'émergence d'opérants verbaux. Nôtre étude a comparé, avec un plan expérimental de sondes multiples concurrents et traitements alternés adapté, l'efficacité du SPOP en comparaison à une procédure de présentation du stimulus contigu avec réponse échoïque dans l'émergence du comportement intraverbal chez trois enfants autistes. Nous avons pu observer l'efficacité du SPOP dans l'induction de l'intraverbal ainsi qu'un rôle facilitateur de l'échoïque. Nous discutons les effets possibles de l'interférence entre traitements, de l'extinction de réponses dans le contexte expérimental, et de l'emploi de renforçateurs conditionnés dans le cadre du SPOP.

Mots-clés : procédure d'observation de paires des stimuli, intraverbal, échoïques, autisme.

\section{Adquisición de intraverbales en niños con autismo: efectos del emparejamiento de estímulos y respuestas ecoicas}

Resumen: La enseñanza de intraverbales a autistas se ha realizado principalmente a través de procedimientos con refuerzo diferencial. Más recientemente, el procedimiento de observación de emparejamiento de estímulo (POEE) surgió como alternativa para verificar la emergencia de repertorios verbales. El POEE implica el emparejamiento de estímulos, sin exigir otra respuesta que la observación del emparejamiento. El ecoico se considera facilitador en la emergencia de operantes verbales. Este estudio comparó, desde un delineamiento de sondas múltiples con tratamiento alternado adaptado, la eficacia del POEE con un procedimiento de presentación de estímulo contiguo solicitante de respuesta ecoica en la emergencia de intraverbales en tres niños autistas. El POEE fue eficaz en la inducción de intraverbales, y el ecoico tuvo un papel facilitador. Se discuten los posibles efectos de interferencia de los tratamientos, de la extinción de respuestas en el contexto experimental y de la utilización de reforzadores condicionados como parte del POEE.

Palabras clave: procedimiento de observación de emparejamiento de estímulos, intraverbales, ecoico, autismo.

\section{Referências}

American Psychiatric Association (2013). Diagnostic and statistical manual of mental disorders (5a ed.). Arlington, VA: American Psychiatric Publishing.

Byrne, B. L., Rehfeldt, R. A., \& Aguirre, A. A. (2014). Evaluating the effectiveness of the stimulus pairing observation procedure and multiple exemplar instruction on tact and listener responses in children with autism. The Analysis of Verbal Behavior, 30(2), 160-169. doi: 10.1007/s40616-014-0020-0

Cao, Y., \& Greer, R. D. (2019). Mastery of echoics in Chinese establishes bidirectional naming in Chinese for preschoolers with naming in English. The Analysis of Verbal Behavior, 34, 79-99. First Online. doi: 10.1007/ s40616-018-0106-1
Carnerero, J. J., \& Pérez-González, L. A. (2014). Induction of naming after observing visual stimuli and their names in children with autism. Research in Developmental Disabilities, 35(10), 2514-2526. doi: 10.1016/j. ridd.2014.06.004

Carnerero, J. J., \& Pérez-González, L. A. (2015). Emergence of naming relations and intraverbals after auditory stimulus pairing. The Psychological Record, 65, 509522. doi: 10.1007/s40732-015-0127-2

Carnerero, J. J., Pérez-González, L. A., \& Osuna, G. (2019). Emergence of naming relations and intraverbals after auditory stimulus pairing: effects of probing the listening skill first. The Psychological Record, 69, 239-252. doi: 10.1007/s40732-019-00336-7 
Coon, J. T., \& Miguel, C. F. (2012). The role of increased exposure to transfer-of-stimulus-control procedures on the acquisition of intraverbal behavior. Journal of Applied Behavior Analysis, 45(4), 657-666. doi: 10.1901/ jaba.2012.45-657

Cortez, M. D., Santos, L., Quintal, A. E., Silveira, M. V., $\&$ de Rose, J. C. (2019). Learning a foreign language: effects of tact and listener instruction on the emergence of bidirectional intraverbals. Journal of Applied Behavior Analysis, 53(1), 484-492. doi: 10.1002/jaba.559

DeCasper, A. J., \& Fifer, W. P. (1980). Of human bonding: newborns prefer their mothers' voices. Science, 208(4448), 1174-1176. doi: 10.1126/ science. 7375928

Eikeseth, S., \& Smith, D. P. (2013). An analysis of verbal stimulus control in intraverbal behavior: implications for practice and applied research. The Analysis of Verbal Behavior, 29, 125-135. doi: 10.1007/bf03393130

Fernald, A. (1993). Approval and disapproval: infant responsiveness to vocal affect in familiar and unfamiliar languages. Child Development, 64(3), 657-674. doi: $10.2307 / 1131209$

Greer, R. D., \& Longano, J. (2010). A rose by naming: how we may learn how to do it. The Analysis of Verbal Behavior, 26, 73-106. doi: 10.1007/BF03393085

Greer, R. D., Pistoljevic, N., Cahill, C., \& Du, L. (2011). Effects of conditioning voices as reinforcers for listener responses on rate of learning, awareness, and preferences for listening to stories in preschoolers with autism. The Analysis of Verbal Behavior, 27, 103-124. doi: 10.1007/ bf03393095

Haggar, J., Ingvarsson, E. T., \& Braun, E. C. (2018). Further evaluation of blocked trials to teach intraverbal responses under complex stimulus control: effects of criterionlevel probes. Learning and Motivation, 62, 29-40. doi: 10.1016/j.lmot.2017.02.006

Horne, P. J., \& Lowe, C. F. (1996). On the origins of naming and other symbolic behavior. Journal of the Experimental Analysis of Behavior, 65(1), 185-241. doi: 10.1901/jeab.1996.65-185

Horner, R. D., \& Baer, D. M. (1978). Multiple-probe technique: a variation of the multiple baseline. Journal of Applied Behavior Analysis, 11(1), 189-196. doi: 10.1901/jaba.1978.11-189

Ingvarsson, E. T., \& Hollobaugh, T. (2011). A comparison of prompting tactics to establish intraverbals in children with autism. Journal of Applied Behavior Analysis, 44(3), 659-664. doi: 10.1901/jaba.2011.44-659

Ingvarsson, E. T., Kramer, R. L., Carp, C. L., Pétursdóttir, A. I., \& Macias, H. (2016). Evaluation of a blockedtrials procedure to establish complex stimulus control over intraverbal responses in children with autism. The Analysis of Verbal Behavior, 32, 205-224. doi: 10.1007/ s40616-016-0071-5

Kisamore, A. N., Karsten, A. M., \& Mann, C. C. (2016). Teaching multiply controlled intraverbals to children and adolescents with autism spectrum disorders. Journal of Applied Behavior Analysis, 49(4), 826-847. doi: 10.1002/jaba.344

Kisamore, A. N., Karsten, A. M., Mann, C. C., \& Conde, K. A. (2013). Effects of a differential observing response on intraverbal performance of preschool children: a preliminary investigation. The Analysis of Verbal Behavior, 29, 101-108. doi: 10.1007/ bf03393127

Kodak, T., Fuchtman, R., \& Paden, A. (2012). A comparison of intraverbal training procedures for children with autism. Journal of Applied Behavior Analysis, 45(1), 155-160. doi: 10.1901/jaba.2012.45-155

Longano, J. M., \& Greer, R. D. (2015). Is the source of reinforcement for naming multiple conditioned reinforcers for observing responses? The Analysis of Verbal Behavior, 31, 96-117. doi: 10.1007/s40616014-0022-y

Lowenkron, B. (1998). Some logical functions of joint control. Journal of the Experimental Analysis of Behavior, 69(3), 327-354. doi: 10.1901/jeab.1998.69-327

Omori, M., \& Yamamoto, J. (2013). Stimulus pairing training for Kanji reading skills in students with developmental disabilities. Research in Developmental Disabilities, 34(4), 1109-1118. doi: 10.1016/j.ridd.2012.12.016

Omori, M., \& Yamamoto, J. (2015). Spelling instruction by stimulus pairing in Japanese students with autism spectrum disorders: effects of stimulus presentation order. The Psychological Record, 65, 401-410. doi: 10.1007/s40732-014-0114-z

Palmer, D. C. (1996). Achieving parity: the role of automatic reinforcement. Journal of the Experimental Analysis of Behavior, 65(1), 289-290. doi: 10.1901/jeab.1996.65-289

Pérez-González, L. A., Cereijo-Blanco, N., \& Carnerero, J. J. (2014). Emerging tacts and selections from previous learned skills: a comparison between two types of naming. The Analysis of Verbal Behavior, 30, 184-192. doi: 10.1007/s40616-014-0011-1

Pérez-González, L. A., García-Conde, A., \& Carnerero, J. J. (2011). Naming completo con estímulos abstractos bidimensionales en niños de seis años. Psicothema, 23(4), 719-724. Recuperado de http://bit.ly/39nuEvz

Pérez-González, L. A., Herszlikowicz, K., \& Williams, G. (2008). Stimulus relations analysis and the emergence of novel intraverbals. The Psychological Record, 58, 95129. doi: 10.1007/BF03395605

Petursdottir, A. I., \& Lepper, T. L. (2015). Inducing novel vocalizations by conditioning speech sounds as reinforcers. Behavior Analysis in Practice, 8, 223-232. doi: 10.1007/s40617-015-0088-6

Petursdottir, A. I., Carp, C. L., Matthies, D. W., \& Esch, B. E. (2011). Analyzing stimulus-stimulus pairing effects on preferences for speech sounds. The Analysis of Verbal Behavior, 27, 45-60. doi: 10.1007/bf03393091

Rosales, R., Rehfeldt, R. A., \& Huffman, N. (2012). Examining the utility of the stimulus pairing observation procedure with preschool children learning a second 
language. Journal of Applied Behavior Analysis, 45(1), 173-177. doi: 10.1901/jaba.2012.45-173

Sindelar, P. T., Rosenberg, M. S., \& Wilson, R. J. (1985). An adapted alternating treatments design for instructional research. Education and Treatment of Children, 8(1), 67-76. Recuperado de http://bit.ly/2wosnln

Skinner, B. F. (1992). Verbal behavior. Acton, MA: Copley. (Trabalho original publicado em 1957).

Stauch, T., LaLonde, K., Plavnick, J. B., Bak, M. Y. S., \& Gatewood, K. (2017). Intraverbal training for individuals with autism: the current status of multiple control. The Analysis of Verbal Behavior, 33, 98-116. doi: 10.1007/ s40616-017-0079-5

Sundberg, M. L. (2014). VB-MAPP: Verbal behavior milestones assessment and placement program. Concord, CA: AVB Press. (Trabalho original publicado em 2008)

Sundberg, M. L., \& Michael, J. (2001). The benefits of Skinner's analysis of verbal behavior for children with autism. Behavior Modification, 25(5), 698-724. doi: 10.1177/0145445501255003

Sundberg, M. L., \& Sundberg, C. A. (2011). Intraverbal behavior and verbal conditional discriminations in typically developing children and children with autism.
The Analysis of Verbal Behavior, 27, 23-44. doi: 10.1007/bf03393090

Takahashi, K., Yamamoto, J., \& Noro, F. (2011). Stimulus pairing training in children with autism spectrum disorder. Research in Autism Spectrum Disorders, 5(1), 547-553. doi: 10.1016/j.rasd.2010.06.021

Vallinger-Brown, M., \& Rosales, R. (2014). An investigation of stimulus pairing and listener training to establish emergent intraverbals in children with autism. The Analysis of Verbal Behavior, 30, 148-159. doi: 10.1007/ s40616-014-0014-y

Vedora, J., \& Conant, E. (2015). A comparison of prompting tactics for teaching intraverbals to young adults with autism. The Analysis of Verbal Behavior, 31, 267-276. doi: 10.1007/s40616-015-0030-6

Vedora, J., Meunier, L., \& Mackay, H. (2009). Teaching intraverbal behavior to children with autism: a comparison of textual and echoic prompts. The Analysis of Verbal Behavior, 25, 79-86. doi: 10.1007/bf03393072

Recebido: $21 / 05 / 2019$

Revisado: 20/02/2020

Aprovado: 26/02/2020 